Lars Allermann

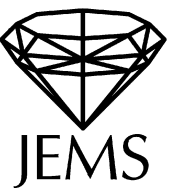

\title{
Tropical intersection products on smooth varieties
}

Received November 22, 2009 and in revised form March 5, 2010

\begin{abstract}
In analogy to [AR07, Chapter 9] we define an intersection product of tropical cycles on tropical linear spaces $L_{k}^{n}$, i.e. on tropical fans of the type $\max \left\{0, x_{1}, \ldots, x_{n}\right\}^{n-k} \cdot \mathbb{R}^{n}$. Afterwards we use this result to obtain an intersection product of cycles on every smooth tropical variety, i.e. on every tropical variety that arises from gluing such tropical linear spaces. In contrast to classical algebraic geometry these products always yield well-defined cycles, not just cycle classes. Using these intersection products we are able to define the pull-back of a tropical cycle along a morphism between smooth tropical varieties. In the present article we stick to the definitions, notions and concepts introduced in [AR07].
\end{abstract}

\section{Intersection products on tropical linear spaces}

In this section we will give a proof that tropical linear spaces $L_{k}^{n}$ admit an intersection product. Therefore we show at first that the diagonal in the Cartesian product $L_{k}^{n} \times L_{k}^{n}$ of such a linear space with itself is a sum of products of Cartier divisors. Given two cycles $C$ and $D$ we can then intersect the diagonal with $C \times D$ and define the product $C \cdot D$ to be the projection thereof.

Throughout the section $e_{1}, \ldots, e_{n}$ will always be the standard basis vectors in $\mathbb{R}^{n}$ and $e_{0}:=-e_{1}-\cdots-e_{n}$.

We begin the section with our basic definitions:

Definition 1.1 (Tropical linear spaces). For $I \subseteq\{0,1, \ldots, n\}$ let $\sigma_{I}$ be the cone generated by the vectors $e_{i}, i \in I$. We denote by $L_{k}^{n}$ the tropical fan consisting of all cones $\sigma_{I}$ with $I \subsetneq\{0,1, \ldots, n\}$ and $|I| \leq k$, whose maximal cones all have weight one (cf. [AR07, Example 3.9]). The fan $L_{k}^{n}$ is a representative of the tropical linear space $\max \left\{0, x_{1}, \ldots, x_{n}\right\}^{n-k} \cdot \mathbb{R}^{n}$.

Definition 1.2. Let $C \in Z_{k}\left(\mathbb{R}^{n}\right)$ be a tropical cycle and let the map $i: \mathbb{R}^{n} \rightarrow \mathbb{R}^{n} \times \mathbb{R}^{n}$ be given by $x \mapsto(x, x)$. Then the push-forward cycle

$$
\triangle_{C}:=i_{*}(C) \in Z_{k}\left(\mathbb{R}^{n} \times \mathbb{R}^{n}\right)
$$

is called the diagonal of $C \times C$.

L. Allermann: Fachbereich Mathematik, TU Kaiserslautern, Postfach 3049, 67653 Kaiserslautern, Germany; e-mail: allerman@mathematik.uni-kl.de 
In order to express the diagonal in $L_{k}^{n} \times L_{k}^{n}$ by means of Cartier divisors we first have to refine $L_{k}^{n} \times L_{k}^{n}$ in such a way that the diagonal is a subfan of this refinement:

Definition 1.3. Let $F_{k}^{n}$ be the refinement of $L_{k}^{n} \times L_{k}^{n}$ that arises recursively from $L_{k}^{n} \times L_{k}^{n}$ as follows: Let $M:=\left(L_{k}^{n} \times L_{k}^{n}\right)^{(2 k)}$ be the set of maximal cones in $L_{k}^{n} \times L_{k}^{n}$. If a cone $\sigma \in M$ is generated by

$$
\left(\frac{-e_{i}}{0}\right),\left(\frac{0}{-e_{i}}\right), v_{3}, \ldots, v_{2 k}
$$

for some $i$ and we have

$$
v_{j} \in\left\{\left(\frac{-e_{\mu}}{-e_{\mu}}\right),\left(\frac{-e_{\mu}}{0}\right),\left(\frac{0}{-e_{\mu}}\right) \mid \mu=0, \ldots, n\right\}
$$

then replace the cone $\sigma$ by the two cones spanned by

$$
\left(\frac{-e_{i}}{-e_{i}}\right),\left(\frac{-e_{i}}{0}\right), v_{3}, \ldots, v_{2 k}
$$

and

$$
\left(\frac{-e_{i}}{-e_{i}}\right),\left(\frac{0}{-e_{i}}\right), v_{3}, \ldots, v_{2 k},
$$

respectively. Repeat this process until there are no more cones in $M$ that can be replaced. The fan $F_{k}^{n}$ is then the set of all faces of all cones in $M$.

The next lemma provides a technical tool needed in the proofs of the subsequent theorems:

Lemma 1.4. Let $F$ be a complete and smooth fan in $\mathbb{R}^{n}$ (in the sense of toric geometry) and let the weight of every maximal cone in $F$ be 1 . Moreover, let $h_{1}, \ldots, h_{r}, r \leq n$, be rational functions on $\mathbb{R}^{n}$ that are linear on every cone of $F$. Then the intersection product $h_{1} \cdots h_{r} \cdot F$ is given by

$$
h_{1} \cdots h_{r} \cdot F=\left(\bigcup_{i=0}^{n-r} F^{(i)}, \omega_{h_{1} \cdots h_{r}}\right)
$$

with some weight function $\omega_{h_{1} \cdots h_{r}}$ on the cones of dimension $n-r$.

Let $\tau \in F^{(n-r)}$ be a cone in $F$ such that for all maximal cones $\sigma \in F^{(n)}$ with $\tau \subseteq \sigma$ there exists some index $i \in\{1, \ldots, r\}$ such that $h_{i}$ is identically zero on $\sigma$. Then

$$
\omega_{h_{1} \cdots h_{r}}(\tau)=0 \text {. }
$$

Proof. We prove the claim by induction on $r$. For $r=1$ we are in the situation that $h_{1}$ is identically zero on every maximal cone adjacent to $\tau$. Hence $\omega_{h_{1}}(\tau)=0$. Now let $r>1$. Using the induction hypothesis we can conclude that $\left|h_{1} \cdots h_{r-1} \cdot F\right| \subseteq \bigcup_{\sigma \in S} \sigma$, where

$$
S:=\left\{\sigma \in F^{(n)} \mid \text { none of } h_{1}, \ldots, h_{r-1} \text { is identically zero on } \sigma\right\} .
$$

Our assumption above then implies that $h_{r}$ must be identically zero on every cone in

$$
\left\{\sigma \in F^{(n)} \mid \tau \subseteq \sigma \text { and none of } h_{1}, \ldots, h_{r-1} \text { is identically zero on } \sigma\right\}
$$

and thus $\omega_{h_{1} \cdots h_{r}}(\tau)=0$. 
Notation 1.5. Let $F$ be a simplicial fan in $\mathbb{R}^{n}$ and let $u$ be a generator of a ray $r_{u}$ in $F$. By abuse of notation we also denote by $u$ the unique rational function on $|F|$ that is linear on every cone in $F$, that has value 1 at $u$ and that is identically zero on all rays of $F$ other than $r_{u}$.

If not stated otherwise, vectors considered as Cartier divisors will from now on always denote rational functions on the complete fan $F_{n}^{n}$.

Notation 1.6. Let $C$ be a tropical cycle and let $h_{1}, \ldots, h_{r} \in \operatorname{Div}(C)$ be Cartier divisors on $C$. If

$$
P\left(x_{1}, \ldots, x_{r}\right)=\sum_{i_{1}+\cdots+i_{r} \leq d} \alpha_{i_{1}, \ldots, i_{r}} x_{1}^{i_{1}} \cdots x_{r}^{i_{r}}
$$

is a polynomial in variables $x_{1}, \ldots, x_{r}$ we denote by $P\left(h_{1}, \ldots, h_{r}\right) \cdot C$ the intersection product

$$
P\left(h_{1}, \ldots, h_{r}\right) \cdot C:=\sum_{i_{1}+\cdots+i_{r} \leq d}\left(\alpha_{i_{1}, \ldots, i_{r}} h_{1}^{i_{1}} \ldots h_{r}^{i_{r}} \cdot C\right) .
$$

In the following theorem we give a description of the diagonal $\triangle_{L_{n-k}^{n}}$ by means of Cartier divisors on our fan $F_{n}^{n}$ :

Theorem 1.7. The fan

$$
\left(\left(\frac{-e_{1}}{0}\right)+\left(\frac{0}{-e_{0}}\right)\right) \cdots\left(\left(\frac{-e_{n}}{0}\right)+\left(\frac{0}{-e_{0}}\right)\right) \cdot\left(\left(\frac{-e_{0}}{0}\right)+\left(\frac{-e_{0}}{-e_{0}}\right)\right)^{k} \cdot F_{n}^{n}
$$

is a representative of the diagonal $\triangle_{L_{n-k}^{n}}$.

Proof. First of all, note that

$$
\left(\frac{-e_{0}}{0}\right)+\left(\frac{-e_{0}}{-e_{0}}\right)
$$

is a representation of the tropical polynomial $\max \left\{0, x_{1}, \ldots, x_{n}\right\}$, where $x_{1}, \ldots, x_{n}$ are the coordinates of the first factor of $\mathbb{R}^{n} \times \mathbb{R}^{n}$. Applying [AR07, Lemma 9.6] we obtain

$$
\left[\left(\left(\frac{-e_{0}}{0}\right)+\left(\frac{-e_{0}}{-e_{0}}\right)\right)^{k} \cdot F_{n}^{n}\right]=\left[L_{n-k}^{n} \times \mathbb{R}^{n}\right]
$$

By [AR07, Lemma 9.4] we conclude that $\triangle_{\mathbb{R}^{n}} \cdot\left[L_{n-k}^{n} \times \mathbb{R}^{n}\right]=i_{*}\left(\left[L_{n-k}^{n}\right]\right)=\triangle_{L_{n-k}^{n}}$ and hence it suffices to show that $[X]=\triangle_{\mathbb{R}^{n}}$ for

$$
X:=\left(\left(\frac{-e_{1}}{0}\right)+\left(\frac{0}{-e_{0}}\right)\right) \cdots\left(\left(\frac{-e_{n}}{0}\right)+\left(\frac{0}{-e_{0}}\right)\right) \cdot F_{n}^{n}
$$

Therefore, let $\sigma=\left\langle r_{1}, \ldots, r_{n}\right\rangle_{\mathbb{R}_{\geq 0}} \in X^{(n)}$ be a cone not contained in $\left|\triangle_{\mathbb{R}^{n}}\right|$. We will show that the weight of $\sigma$ in $X$ has to be zero. W.l.o.g. we can assume that

$$
r_{1} \notin D:=\left\{\left(\frac{-e_{0}}{-e_{0}}\right), \ldots,\left(\frac{-e_{n}}{-e_{n}}\right)\right\} .
$$


Moreover, let

$$
T:=\left\{\left(\frac{-e_{1}}{0}\right), \ldots,\left(\frac{-e_{n}}{0}\right)\right\} \quad \text { and } \quad B:=\left\{\left(\frac{0}{-e_{1}}\right), \ldots,\left(\frac{0}{-e_{n}}\right)\right\} .
$$

We distinguish two cases:

Case 1. First, we assume that

$$
r_{i} \notin\left\{\left(\frac{-e_{0}}{0}\right),\left(\frac{0}{-e_{0}}\right)\right\}, \quad i=1, \ldots, n .
$$

By adding globally linear functions to the given rational functions we can rewrite the above intersection product as $X=\varphi_{1} \cdots \varphi_{n} \cdot F_{n}^{n}$, where

$$
\varphi_{i}= \begin{cases}\left(\frac{-e_{i}}{0}\right)+\left(\frac{0}{-e_{0}}\right) & \text { if }\left(\frac{-e_{i}}{0}\right) \notin\left\{r_{1}, \ldots, r_{n}\right\} \\ \left(\frac{0}{-e_{i}}\right)+\left(\frac{-e_{0}}{0}\right) & \text { else. }\end{cases}
$$

Now we apply Lemma 1.4: If the weight of $\sigma$ in $X$ is non-zero there must be at least one cone

$$
\widetilde{\sigma}=\left\langle r_{1}, \ldots, r_{n}, v_{1}, \ldots, v_{n}\right\rangle_{\mathbb{R} \geq 0} \in F_{n}^{n}
$$

such that all rational functions $\varphi_{1}, \ldots, \varphi_{n}$ are non-zero on $\widetilde{\sigma}$. We study three subcases:

(a) There are vectors $r_{i} \in T$ and $r_{j} \in B$ : Then we need to have both vectors $\left(\frac{-e_{0}}{0}\right)$ and $\left(\frac{0}{-e_{0}}\right)$ among the $v_{\mu}$ in order that all functions $\varphi_{i}$ be non-zero on $\tilde{\sigma}$. But there is no cone in $F_{n}^{n}$ containing these two vectors.

(b) $r_{1} \in T$ (or $r_{1} \in B$ ) and $r_{j} \in D$ for some $j$ and $r_{i} \in T \cup D$ (or $r_{i} \in B \cup D$ ) for all $i$ : As there is no cone in $F$ containing $\left(\frac{-e_{i}}{0}\right)$ and $\left(\frac{0}{-e_{i}}\right)$ for any $i$, we need $\left(\frac{-e_{0}}{0}\right)$ among the $v_{\mu}$ to have all $\varphi_{i}$ non-zero on $\widetilde{\sigma}$. Moreover, if $\left(\frac{-e_{i}}{0}\right) \notin\left\{r_{1}, \ldots, r_{n}\right\}$ then we must have $\left(\frac{-e_{i}}{0}\right) \in\left\{v_{1}, \ldots, v_{n}\right\}$. But there is no cone in $F_{n}^{n}$ containing $\left(\frac{-e_{1}}{0}\right), \ldots,\left(\frac{-e_{n}}{0}\right)$ and $\left(\frac{-e_{0}}{0}\right)$. (Analogously for $B$, but with $\varphi_{i}$ defined the other way around.)

(c) All vectors $r_{i}$ are contained in $T$ (or in $B$ ): In this case we need $\left(\frac{0}{-e_{1}}\right)$ or $\left(\frac{-e_{0}}{0}\right)$ among the $v_{\mu}$ to have all functions $\varphi_{i}$ non-zero, but again there is no such cone. (Analogously for $B$, but with $\varphi_{i}$ defined the other way around.)

Case 2. Now we assume that

$$
r_{1}=\left(\frac{-e_{0}}{0}\right)\left(\text { or } r_{1}=\left(\frac{0}{-e_{0}}\right)\right)
$$

As before we rewrite the intersection product as $X=\varphi_{1} \cdots \varphi_{n} \cdot F_{n}^{n}$ with $\varphi_{i}$ defined as above and apply Lemma 1.4: If $\left(\frac{-e_{i}}{0}\right) \notin\left\{r_{1}, \ldots, r_{n}\right\}$ then $\varphi_{i}=\left(\frac{-e_{i}}{0}\right)+\left(\frac{0}{-e_{0}}\right)$ and we 
need $\left(\frac{-e_{i}}{0}\right)$ or $\left(\frac{0}{-e_{0}}\right)$ among the $v_{\mu}$ to have all $\varphi_{i}$ non-zero on $\widetilde{\sigma}$. But as there is no cone in $F_{n}^{n}$ containing $\left(\frac{0}{-e_{0}}\right)$ and $\left(\frac{-e_{0}}{0}\right)$ we must have $\left(\frac{-e_{i}}{0}\right) \in\left\{v_{1}, \ldots, v_{n}\right\}$. Hence all the vectors $\left(\frac{-e_{1}}{0}\right), \ldots,\left(\frac{-e_{n}}{0}\right)$ and $\left(\frac{-e_{0}}{0}\right)$ must be contained in $\left\{r_{1}, \ldots, r_{n}, v_{1}, \ldots, v_{n}\right\}$; but there is no such cone in $F_{n}^{n}$. (Analogously for $r_{1}=\left(\frac{0}{-e_{0}}\right)$, but with $\varphi_{i}$ defined the other way around.)

So far we have proven that our intersection cycle $X$ is contained in the diagonal $\triangle_{\mathbb{R}^{n}}$. As the diagonal is irreducible we can then conclude by [GKM07, Lemma 2.21] that $[X]=$ $\lambda \cdot \triangle_{\mathbb{R}^{n}}$ for some integer $\lambda$. Thus our last step in this proof is to show that $\lambda=1$ : Let $\varphi_{1}, \ldots, \varphi_{n}$ be the rational functions given above. We obtain the following equality of cycles in $\mathbb{R}^{n} \times \mathbb{R}^{n}$ :

$$
\begin{aligned}
\varphi_{1} \cdots \varphi_{n} \cdot\left[\{0\} \times \mathbb{R}^{n}\right] & =\left(\left(\frac{-e_{1}}{0}\right)+\left(\frac{0}{-e_{0}}\right)\right) \cdots\left(\left(\frac{-e_{n}}{0}\right)+\left(\frac{0}{-e_{0}}\right)\right) \cdot\left[\{0\} \times \mathbb{R}^{n}\right] \\
& =\left(\frac{0}{-e_{0}}\right)^{n} \cdot\left[\{0\} \times \mathbb{R}^{n}\right]=\{0\} \times\{0\} .
\end{aligned}
$$

As $\varphi_{1} \cdots \varphi_{n} \cdot\left[\mathbb{R}^{n} \times \mathbb{R}^{n}\right]=\lambda \cdot \triangle_{\mathbb{R}^{n}}$, by [AR07, Definition 9.3 and Remark 9.9] we obtain the equality

$$
\left.\lambda \cdot\{0\}=\lambda \cdot\left(\{0\} \cdot \mathbb{R}^{n}\right)=\pi_{*}\left(\varphi_{1} \cdots \varphi_{n} \cdot\left(\{0\} \times \mathbb{R}^{n}\right)\right)=\pi_{*}(\{0\} \times\{0\})\right)=1 \cdot\{0\}
$$

of cycles in $\mathbb{R}^{n}$. This finishes the proof.

Our next step is to derive a description of the diagonal $\triangle_{L_{n-k}^{n}}$ on $L_{n-k}^{n} \times L_{n-k}^{n}$ from our description on $F_{n}^{n}$ :

Theorem 1.8. The intersection product in Theorem 1.7 can be rewritten as

$$
\left(\sum_{i=1}^{r} h_{i, 1} \cdots h_{i, n-k}\right) \cdot\left(\left(\frac{0}{-e_{0}}\right)+\left(\frac{-e_{0}}{-e_{0}}\right)\right)^{k} \cdot\left(\left(\frac{-e_{0}}{0}\right)+\left(\frac{-e_{0}}{-e_{0}}\right)\right)^{k} \cdot F_{n}^{n}
$$

for some Cartier divisors $h_{i, j}$ on $F_{n}^{n}$.

We prepare the proof of the theorem by the following lemma:

Lemma 1.9. Let $C \in Z_{l}\left(L_{n-k}^{n}\right)$ be a subcycle of $L_{n-k}^{n}$. Then the following intersection products are zero:
(a) $\left(\frac{-e_{0}}{0}\right) \cdot\left(\frac{0}{-e_{0}}\right) \cdot\left(C \times \mathbb{R}^{n}\right)$,
(b) $v_{i_{1}} \cdots v_{i_{n-k+r}} \cdot\left(C \times \mathbb{R}^{n}\right)$,
(c) $\left(\frac{0}{-e_{0}}\right) \cdot\left(\frac{-e_{0}}{-e_{0}}\right)^{s} \cdot v_{i_{1}} \cdots v_{i_{n-k-s+r}} \cdot\left(C \times \mathbb{R}^{n}\right)$, 
where $r, s>0$ and the vectors

$$
v_{i_{j}} \in\left\{\left(\frac{-e_{1}}{0}\right), \ldots,\left(\frac{-e_{n}}{0}\right),\left(\frac{-e_{0}}{-e_{0}}\right)\right\}
$$

are pairwise distinct.

Proof. (a)\&(b) In both cases, a cone that can occur in the intersection product with nonzero weight has to be contained in a cone of $F_{n}^{n}$ that is contained in $\left|L_{n-k}^{n} \times \mathbb{R}^{n}\right|$ and that contains the vectors $\left(\frac{-e_{0}}{0}\right),\left(\frac{0}{-e_{0}}\right)$ or $v_{i_{1}}, \ldots, v_{i_{n-k+r}}$, respectively. But there are no such cones.

(c) By (a) and [AR07, Lemma 9.7] we can rewrite the intersection product as

$$
\begin{aligned}
\left(\frac{0}{-e_{0}}\right) \cdot\left(\frac{-e_{0}}{-e_{0}}\right)^{s} \cdot v_{i_{1}} \cdots v_{i_{n-k-s+r}} \cdot\left(C \times \mathbb{R}^{n}\right) & \\
= & \left(\frac{0}{-e_{0}}\right) \cdot\left(\left(\frac{-e_{0}}{0}\right)+\left(\frac{-e_{0}}{-e_{0}}\right)\right)^{s} \cdot v_{i_{1}} \cdots v_{i_{n-k-s+r}} \cdot\left(C \times \mathbb{R}^{n}\right) \\
& =\left(\frac{0}{-e_{0}}\right) \cdot v_{i_{1}} \cdots v_{i_{n-k-s+r}} \cdot\left[\left(\left(\frac{-e_{0}}{0}\right)+\left(\frac{-e_{0}}{-e_{0}}\right)\right)^{s} \cdot C\right] \times \mathbb{R}^{n} \\
& =\left(\frac{0}{-e_{0}}\right) \cdot v_{i_{1}} \cdots v_{i_{n-k-s+r}} \cdot\left[\max \left\{0, x_{1}, \ldots, x_{n}\right\}^{s} \cdot C\right] \times \mathbb{R}^{n},
\end{aligned}
$$

which is zero by (b) as $\max \left\{0, x_{1}, \ldots, x_{n}\right\}^{s} \cdot C$ is contained in $L_{n-k-s}^{n}$.

Proof of Theorem 1.8. By Theorem 1.7 we have the representation

$$
\begin{aligned}
\triangle_{L_{n-k}^{n}}= & \left(\left(\frac{-e_{1}}{0}\right)+\left(\frac{0}{-e_{0}}\right)\right) \cdots\left(\left(\frac{-e_{n}}{0}\right)+\left(\frac{0}{-e_{0}}\right)\right) \\
& \cdot \underbrace{\left(\left(\frac{-e_{0}}{0}\right)+\left(\frac{-e_{0}}{-e_{0}}\right)\right)^{k} \cdot\left[F_{n}^{n}\right]}_{=\left[L_{n-k}^{n} \times \mathbb{R}^{n}\right]} \\
= & \left(\left(\frac{-e_{1}}{0}\right) \cdots\left(\frac{-e_{n}}{0}\right)+\cdots+\left(\frac{0}{-e_{0}}\right)^{n}\right) \cdot\left[L_{n-k}^{n} \times \mathbb{R}^{n}\right] .
\end{aligned}
$$

By Lemma 1.9(b) all the summands containing $\left(\frac{0}{-e_{0}}\right)^{s}$ with $s<k$ are zero. Hence we can rewrite the intersection product as

$$
\begin{aligned}
\triangle_{L_{n-k}^{n}}= & {\left[\left(\frac{-e_{1}}{0}\right) \cdots\left(\frac{-e_{n-k}}{0}\right)+\cdots+\left(\frac{0}{-e_{0}}\right)^{n-k} \cdot\left(\left(\frac{0}{-e_{0}}\right)+\left(\frac{-e_{0}}{-e_{0}}\right)\right)^{k}-A\right] } \\
& \cdot\left[L_{n-k}^{n} \times \mathbb{R}^{n}\right],
\end{aligned}
$$

where $A$ contains all the summands we added too much. Thus all the summands of $A$ are of the form

$$
\alpha \cdot v_{1} \cdots v_{n-s-t} \cdot\left(\frac{0}{-e_{0}}\right)^{s} \cdot\left(\frac{-e_{0}}{-e_{0}}\right)^{t}
$$


for some integer $\alpha$, vectors $v_{i} \in\left\{\left(\frac{-e_{1}}{0}\right), \ldots,\left(\frac{-e_{n}}{0}\right)\right\}$ and powers $1 \leq t \leq k, 0 \leq s \leq n$. By Lemma 1.9(b)\&(c) such a summand applied to $\left[L_{n-k}^{n} \times \mathbb{R}^{n}\right]$ is zero if $s<k$ and only those summands remain in $A$ that have $t \geq 1, s \geq k$. Let

$$
S:=\alpha \cdot v_{1} \cdots v_{n-s-t} \cdot\left(\frac{0}{-e_{0}}\right)^{s} \cdot\left(\frac{-e_{0}}{-e_{0}}\right)^{t}
$$

be one of the remaining summands. By Lemma 1.9(a) we obtain

$$
\begin{aligned}
& \alpha \cdot v_{1} \cdots v_{n-s-t} \cdot\left(\frac{0}{-e_{0}}\right)^{s} \cdot\left(\frac{-e_{0}}{-e_{0}}\right)^{t} \cdot\left[L_{n-k}^{n} \times \mathbb{R}^{n}\right] \\
&=\left(\sum_{j=0}^{t}\left(\begin{array}{l}
t \\
j
\end{array}\right) \cdot \alpha \cdot v_{1} \cdots v_{n-s-t} \cdot\left(\frac{0}{-e_{0}}\right)^{s} \cdot\left(\frac{-e_{0}}{-e_{0}}\right)^{j} \cdot\left(\frac{-e_{0}}{0}\right)^{t-j}\right) \cdot\left[L_{n-k}^{n} \times \mathbb{R}^{n}\right] \\
&=\left(\alpha \cdot v_{1} \cdots v_{n-s-t} \cdot\left(\frac{0}{-e_{0}}\right)^{s} \cdot\left(\left(\frac{-e_{0}}{0}\right)+\left(\frac{-e_{0}}{-e_{0}}\right)\right)^{t}\right) \cdot\left[L_{n-k}^{n} \times \mathbb{R}^{n}\right] \\
&= {\left[\left(\left(\frac{0}{-e_{0}}\right)+\left(\frac{-e_{0}}{-e_{0}}\right)\right)^{k}\right.} \\
&\left.\cdot\left(\alpha \cdot v_{1} \cdots v_{n-s-t} \cdot\left(\frac{0}{-e_{0}}\right)^{s-k} \cdot\left(\left(\frac{-e_{0}}{0}\right)+\left(\frac{-e_{0}}{-e_{0}}\right)\right)^{t}\right)-B_{S}\right] \cdot\left[L_{n-k}^{n} \times \mathbb{R}^{n}\right],
\end{aligned}
$$

where $B_{S}$ again contains all the summands we added too much. Thus all the summands of $B_{S}$ are of the form

$$
S^{\prime}:=\beta \cdot\left(\begin{array}{c}
t \\
t^{\prime}
\end{array}\right) \cdot v_{1} \cdots v_{n-s-t} \cdot\left(\frac{0}{-e_{0}}\right)^{s-s^{\prime}} \cdot\left(\frac{-e_{0}}{-e_{0}}\right)^{s^{\prime}} \cdot\left(\frac{-e_{0}}{0}\right)^{t^{\prime}} \cdot\left(\frac{-e_{0}}{-e_{0}}\right)^{t-t^{\prime}}
$$

for some integer $\beta$ and powers $1 \leq s^{\prime} \leq k, 0 \leq t^{\prime} \leq t$. If $s-s^{\prime}<k$ we group all corresponding summands together as

$$
\beta \cdot v_{1} \cdots v_{n-s-t} \cdot\left(\frac{0}{-e_{0}}\right)^{s-s^{\prime}} \cdot\left(\frac{-e_{0}}{-e_{0}}\right)^{s^{\prime}} \cdot\left(\left(\frac{-e_{0}}{0}\right)+\left(\frac{-e_{0}}{-e_{0}}\right)\right)^{t} .
$$

This product applied to $\left[L_{n-k}^{n} \times \mathbb{R}^{n}\right]$ is zero by Lemma 1.9(b)\&(c). Moreover, all summands $S^{\prime}$ with $s-s^{\prime} \geq k$ and $t^{\prime}>0$ yield zero on $\left[L_{n-k}^{n} \times \mathbb{R}^{n}\right]$ by Lemma 1.9(a). Thus only those summands $S^{\prime}$ are left in $B_{S}$ that are of the form

$$
S^{\prime}=\beta^{\prime} \cdot v_{1} \cdots v_{n-s-t} \cdot\left(\frac{0}{-e_{0}}\right)^{s-s^{\prime}} \cdot\left(\frac{-e_{0}}{-e_{0}}\right)^{t+s^{\prime}}
$$

with $s-s^{\prime} \geq k$ and $s^{\prime} \geq 1$. Applying this process inductively to all summands with $t=1, \ldots, n-k-1$ in which we could not factor out $\left(\left(\frac{0}{-e_{0}}\right)+\left(\frac{-e_{0}}{-e_{0}}\right)\right)^{k}$ yet, we can 
by and by increase the power of $\left(\frac{-e_{0}}{-e_{0}}\right)$ in all remaining summands until finally only one summand

$$
\gamma \cdot\left(\frac{0}{-e_{0}}\right)^{k} \cdot\left(\frac{-e_{0}}{-e_{0}}\right)^{n-k}
$$

is left. But

$$
\begin{aligned}
& \gamma \cdot\left(\frac{0}{-e_{0}}\right)^{k} \cdot\left(\frac{-e_{0}}{-e_{0}}\right)^{n-k} \cdot\left[L_{n-k}^{n} \times \mathbb{R}^{n}\right] \\
& \quad=\gamma \cdot\left(\left(\frac{0}{-e_{0}}\right)+\left(\frac{-e_{0}}{-e_{0}}\right)\right)^{k} \cdot\left(\left(\frac{-e_{0}}{0}\right)+\left(\frac{-e_{0}}{-e_{0}}\right)\right)^{n-k} \cdot\left[L_{n-k}^{n} \times \mathbb{R}^{n}\right]
\end{aligned}
$$

as

$$
\begin{aligned}
\left(\frac{0}{-e_{0}}\right)^{i} \cdot\left(\frac{-e_{0}}{-e_{0}}\right)^{k-i} \cdot\left(\left(\frac{-e_{0}}{0}\right)+\left(\frac{-e_{0}}{-e_{0}}\right)\right)^{n-k} \cdot\left[L_{n-k}^{n} \times \mathbb{R}^{n}\right] & \\
& =\left(\frac{0}{-e_{0}}\right)^{i} \cdot\left(\frac{-e_{0}}{-e_{0}}\right)^{k-i} \cdot\left[L_{0}^{n} \times \mathbb{R}^{n}\right]=0
\end{aligned}
$$

for all $i<k$ by Lemma 1.9(b) and

$$
\left(\frac{0}{-e_{0}}\right)^{k} \cdot\left(\frac{-e_{0}}{0}\right)^{j} \cdot\left(\frac{-e_{0}}{-e_{0}}\right)^{n-k-j} \cdot\left[L_{n-k}^{n} \times \mathbb{R}^{n}\right]=0
$$

for all $j>0$ by Lemma 1.9(a). This proves the claim.

Example 1.10. We perform the steps described in the proof of Theorem 1.8 for the case $n=3, k=2$ : By Theorem 1.7 we have the representation

$$
\begin{aligned}
\triangle_{L_{1}^{3}}= & \left(\left(\frac{-e_{1}}{0}\right)+\left(\frac{0}{-e_{0}}\right)\right) \cdot\left(\left(\frac{-e_{2}}{0}\right)+\left(\frac{0}{-e_{0}}\right)\right) \cdot\left(\left(\frac{-e_{3}}{0}\right)+\left(\frac{0}{-e_{0}}\right)\right) \\
& \cdot \underbrace{\left(\left(\frac{-e_{0}}{0}\right)+\left(\frac{-e_{0}}{-e_{0}}\right)\right)^{2} \cdot\left[F_{3}^{3}\right]}_{=\left[L_{1}^{3} \times \mathbb{R}^{3}\right]} \\
= & (\underbrace{\left(\frac{-e_{1}}{0}\right) \cdot\left(\frac{-e_{2}}{0}\right) \cdot\left(\frac{-e_{3}}{0}\right)}_{=0 \text { by Lemma 1.9(b) }}+\underbrace{\left(\frac{-e_{1}}{0}\right) \cdot\left(\frac{-e_{2}}{0}\right) \cdot\left(\frac{0}{-e_{0}}\right)}_{=0 \text { by Lemma 1.9(b) }} \\
& +\underbrace{\left(\frac{-e_{1}}{0}\right) \cdot\left(\frac{-e_{3}}{0}\right) \cdot\left(\frac{0}{-e_{0}}\right)}_{=0 \text { by Lemma 1.9(b) }}+\underbrace{\left(\frac{-e_{2}}{0}\right) \cdot\left(\frac{-e_{3}}{0}\right) \cdot\left(\frac{0}{-e_{0}}\right)}_{=0 \text { by Lemma 1.9(b) }} \\
& \left.+\left(\frac{-e_{1}}{0}\right) \cdot\left(\frac{0}{-e_{0}}\right)^{2}+\left(\frac{-e_{2}}{0}\right) \cdot\left(\frac{-e_{3}}{0}\right)^{2} \cdot\left(\frac{0}{-e_{0}}\right)^{2}+\left(\frac{0}{-e_{0}}\right)^{3}\right) \\
& \cdot\left[L_{1}^{3} \times \mathbb{R}^{3}\right] .
\end{aligned}
$$


Now we factor out $\left(\left(\frac{0}{-e_{0}}\right)+\left(\frac{-e_{0}}{-e_{0}}\right)\right)^{2}$ and subtract all summands we do not need:

$$
\begin{aligned}
\triangle_{L_{1}^{3}}= & \left(\left(\frac{-e_{1}}{0}\right)+\left(\frac{-e_{2}}{0}\right)+\left(\frac{-e_{3}}{0}\right)+\left(\frac{0}{-e_{0}}\right)\right) \cdot\left(\left(\frac{0}{-e_{0}}\right)+\left(\frac{-e_{0}}{-e_{0}}\right)\right)^{2} \cdot\left[L_{1}^{3} \times \mathbb{R}^{3}\right] \\
& -(\underbrace{\left(\frac{-e_{1}}{0}\right)\left(\frac{-e_{0}}{-e_{0}}\right)^{2}}_{=0 \text { by } 1.9(\mathrm{~b})}+\underbrace{\left(\frac{-e_{2}}{0}\right)\left(\frac{-e_{0}}{-e_{0}}\right)^{2}}_{=0 \text { by } 1.9(\mathrm{~b})}+\underbrace{\left(\frac{-e_{3}}{0}\right)\left(\frac{-e_{0}}{-e_{0}}\right)^{2}}_{=0 \text { by } 1.9(\mathrm{~b})} \\
& +\underbrace{\left(\frac{0}{-e_{0}}\right)\left(\frac{-e_{0}}{-e_{0}}\right)^{2}}_{=0 \text { by } 1.9(\mathrm{~b})}+\underbrace{2\left(\frac{-e_{1}}{0}\right)\left(\frac{0}{-e_{0}}\right)\left(\frac{-e_{0}}{-e_{0}}\right)}_{=0 \text { by } 1.9(\mathrm{c})}+\underbrace{2\left(\frac{-e_{2}}{0}\right)\left(\frac{0}{-e_{0}}\right)\left(\frac{-e_{0}}{-e_{0}}\right)}_{=0 \text { by } 1.9(\mathrm{~b})} \\
& +\underbrace{2\left(\frac{-e_{3}}{0}\right)\left(\frac{0}{-e_{0}}\right)\left(\frac{-e_{0}}{-e_{0}}\right)}+2\left(\frac{0}{-e_{0}}\right)^{2}\left(\frac{-e_{0}}{-e_{0}}\right)) \cdot\left[L_{1}^{3} \times \mathbb{R}^{3}\right] .
\end{aligned}
$$

But by Lemma 1.9(a)\&(b) we have the following equation for the last summand:

$$
\begin{aligned}
& -2\left(\frac{0}{-e_{0}}\right)^{2}\left(\frac{-e_{0}}{-e_{0}}\right) \cdot\left[L_{1}^{3} \times \mathbb{R}^{3}\right] \\
& =-2\left(\left(\frac{0}{-e_{0}}\right)^{2}+2\left(\frac{0}{-e_{0}}\right)\left(\frac{-e_{0}}{-e_{0}}\right)+\left(\frac{-e_{0}}{-e_{0}}\right)^{2}\right) \cdot\left(\left(\frac{-e_{0}}{0}\right)+\left(\frac{-e_{0}}{-e_{0}}\right)\right) \cdot\left[L_{1}^{3} \times \mathbb{R}^{3}\right] .
\end{aligned}
$$

Hence altogether we obtain

$$
\begin{aligned}
\triangle_{L_{1}^{3}}= & \left(\left(\frac{-e_{1}}{0}\right)+\left(\frac{-e_{2}}{0}\right)+\left(\frac{-e_{3}}{0}\right)+\left(\frac{0}{-e_{0}}\right)-2\left(\frac{-e_{0}}{0}\right)-2\left(\frac{-e_{0}}{-e_{0}}\right)\right) \\
& \cdot\left(\left(\frac{0}{-e_{0}}\right)+\left(\frac{-e_{0}}{-e_{0}}\right)\right)^{2} \cdot\left(\left(\frac{-e_{0}}{0}\right)+\left(\frac{-e_{0}}{-e_{0}}\right)\right)^{2} \cdot\left[\mathbb{R}^{3} \times \mathbb{R}^{3}\right] .
\end{aligned}
$$

Corollary 1.11. The Cartier divisors $h_{i, j}$ from Theorem 1.8 provide the following description of the diagonal $\triangle_{L_{n-k}^{n}}$ :

$$
\triangle_{L_{n-k}^{n}}=\sum_{i=1}^{r} h_{i, 1} \cdots h_{i, n-k} \cdot\left[L_{n-k}^{n} \times L_{n-k}^{n}\right] .
$$

Proof. Let $x_{1}, \ldots, x_{n}$ be the coordinates of the first factor and $y_{1}, \ldots, y_{n}$ the coordinates of the second factor of $\mathbb{R}^{n} \times \mathbb{R}^{n}$. Applying [AR07, Lemma 9.6] we conclude that

$$
\begin{aligned}
{\left[\left(\left(\frac{0}{-e_{0}}\right)\right.\right.} & \left.\left.+\left(\frac{-e_{0}}{-e_{0}}\right)\right)^{k} \cdot\left(\left(\frac{-e_{0}}{0}\right)+\left(\frac{-e_{0}}{-e_{0}}\right)\right)^{k} \cdot F_{n}^{n}\right] \\
& =\left[\max \left\{0, x_{1}, \ldots, x_{n}\right\}^{k} \cdot \max \left\{0, y_{1}, \ldots, y_{n}\right\}^{k} \cdot F_{n}^{n}\right]=\left[L_{n-k}^{n} \times L_{n-k}^{n}\right]
\end{aligned}
$$


and hence by Theorems 1.7 and 1.8 ,

$$
\sum_{i=1}^{r} h_{i, 1} \cdots h_{i, n-k} \cdot\left[L_{n-k}^{n} \times L_{n-k}^{n}\right]=\triangle_{L_{n-k}^{n}}
$$

Remark 1.12. As Lemma 1.9 does not only hold on $L_{n-k}^{n} \times \mathbb{R}^{n}$ but also on any $C \times \mathbb{R}^{n}$ with $C$ a subcycle of $L_{n-k}^{n}$, the proof of Theorem 1.8 indeed shows that

$$
\begin{aligned}
\left(\sum_{i=1}^{r} h_{i, 1} \cdots h_{i, n-k}\right) & \left(\left(\frac{0}{-e_{0}}\right)+\left(\frac{-e_{0}}{-e_{0}}\right)\right)^{k} \cdot\left(C \times \mathbb{R}^{n}\right) \\
= & \left(\left(\frac{-e_{1}}{0}\right)+\left(\frac{0}{-e_{0}}\right)\right) \cdots\left(\left(\frac{-e_{n}}{0}\right)+\left(\frac{0}{-e_{0}}\right)\right) \cdot\left(C \times \mathbb{R}^{n}\right)
\end{aligned}
$$

for all cycles $C \in Z_{l}\left(L_{n-k}^{n}\right)$. Using [AR07, Corollary 9.8] we conclude that

$$
\left(\sum_{i=1}^{r} h_{i, 1} \cdots h_{i, n-k}\right) \cdot\left(\left(\frac{0}{-e_{0}}\right)+\left(\frac{-e_{0}}{-e_{0}}\right)\right)^{k} \cdot\left(C \times \mathbb{R}^{n}\right)=\triangle_{\mathbb{R}^{n}} \cdot\left(C \times \mathbb{R}^{n}\right)=\triangle_{C}
$$

for all such cycles $C$.

Corollary 1.13. Let $\sigma \in L_{n-k}^{n}$, let $x \in \sigma$ and let $U \subseteq S_{\sigma}=\bigcup_{\sigma^{\prime} \in L_{n-k}^{n}: \sigma^{\prime} \supseteq \sigma}\left(\sigma^{\prime}\right)^{r i}$ be an open subset of $\left|L_{n-k}^{n}\right|$ containing $x$. Moreover, let $F$ be the open fan $F:=\{-x+\sigma \cap U \mid$ $\left.\sigma \in L_{n-k}^{n}\right\}$ and $\widetilde{F}$ the associated tropical fan. Then there are Cartier divisors $h_{i, j}^{\prime}$ on $\widetilde{F} \times \widetilde{F}$ such that

$$
\triangle_{[\widetilde{F}]}=\sum_{i=1}^{r} h_{i, 1}^{\prime} \cdots h_{i, n-k}^{\prime} \cdot[\widetilde{F} \times \widetilde{F}] .
$$

Proof. To obtain the Cartier divisors $h_{i, j}^{\prime}$ we just have to restrict the Cartier divisors $h_{i, j}$ from Corollary 1.11 to the open set $U \times U_{\mathcal{\sim}}$ translate them suitably and extend them from

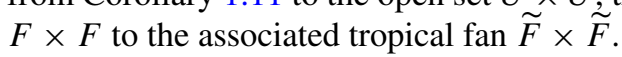

Example 1.14. The following figure shows two fans associated to open subsets of $L_{2}^{3}$ as in Corollary 1.13:

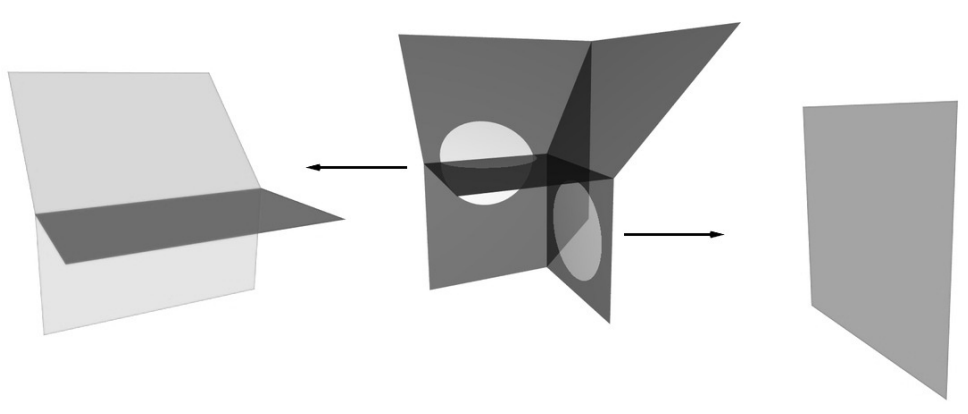


Lemma 1.15. Let $C \in Z_{k}\left(\mathbb{R}^{n}\right)$ and $D \in Z_{l}\left(\mathbb{R}^{n}\right)$ be tropical cycles such that there exist representations of the diagonals $\triangle_{C}$ and $\triangle_{D}$ as sums of products of Cartier divisors on $C \times C$ and $D \times D$, respectively. Then there also exists a representation of $\triangle_{C \times D}$ as a sum of products of Cartier divisors on $(C \times D)^{2}$.

Proof. Let $\triangle_{C}=\sum_{i=1}^{r} \varphi_{i, 1} \cdots \varphi_{i, k} \cdot(C \times C)$ and $\triangle_{D}=\sum_{i=1}^{s} \psi_{i, 1} \cdots \psi_{i, l} \cdot(D \times D)$. Moreover, let $\pi_{x}, \pi_{y}:\left(\mathbb{R}^{n}\right)^{4} \rightarrow\left(\mathbb{R}^{n}\right)^{2}$ be given by $\left(x_{1}, y_{1}, x_{2}, y_{2}\right) \mapsto\left(x_{1}, x_{2}\right)$ and $\left(x_{1}, y_{1}, x_{2}, y_{2}\right) \mapsto\left(y_{1}, y_{2}\right)$, respectively. Then

$$
\triangle_{C \times D}=\left(\sum_{i=1}^{r} \pi_{x}^{*} \varphi_{i, 1} \cdots \pi_{x}^{*} \varphi_{i, k}\right) \cdot\left(\sum_{i=1}^{s} \pi_{y}^{*} \psi_{i, 1} \cdots \pi_{y}^{*} \psi_{i, l}\right) \cdot(C \times D)^{2} .
$$

Now we are ready to define intersection products on all spaces on which we can express the diagonal by means of Cartier divisors:

Definition 1.16 (Intersection products). Let $C \in Z_{k}\left(\mathbb{R}^{n}\right)$ be a tropical cycle and assume that there are Cartier divisors $\varphi_{i, j}$ on $C \times C$ such that

$$
\triangle_{C}=\sum_{i=1}^{r} \varphi_{i, 1} \cdots \varphi_{i, k} \cdot(C \times C) .
$$

Moreover, let $\pi: C \times C \rightarrow C$ be the morphism given by $(x, y) \mapsto x$. Then we define the intersection product of subcycles of $C$ by

$$
\begin{aligned}
Z_{k-l}(C) \times Z_{k-l^{\prime}}(C) & \rightarrow Z_{k-l-l^{\prime}}(C), \\
\left(D_{1}, D_{2}\right) & \mapsto D_{1} \cdot D_{2}:=\pi_{*}\left(\sum_{i=1}^{r} \varphi_{i, 1} \cdots \varphi_{i, k} \cdot\left(D_{1} \times D_{2}\right)\right) .
\end{aligned}
$$

We use the rest of this section to prove that this intersection product is independent of the representation of the diagonal used and that it has all the properties we expect-at least for those spaces we are interested in:

Lemma 1.17. Let $C \in Z_{k}\left(\mathbb{R}^{n}\right)$ be a tropical cycle, $D \in Z_{k-l}(C), E \in Z_{k-l^{\prime}}(C)$ be subcycles, let $\varphi \in \operatorname{Div}(C)$ be a Cartier divisor and $\pi: C \times C \rightarrow C$ the morphism given by $(x, y) \mapsto x$. Then

$$
(\varphi \cdot D) \times E=\pi^{*} \varphi \cdot(D \times E) .
$$

Proof. The proof is exactly the same as for [AR07, Lemma 9.6].

Corollary 1.18. Let $C \in Z_{k}\left(\mathbb{R}^{n}\right)$ be a tropical cycle that admits an intersection product as in Definition 1.16, let $D \in Z_{k-l}(C), E \in Z_{k-l^{\prime}}(C)$ be subcycles and let $\varphi \in \operatorname{Div}(C)$ be a Cartier divisor. Then

$$
(\varphi \cdot D) \cdot E=\varphi \cdot(D \cdot E) .
$$

Proof. The proof is exactly the same as for [AR07, Lemma 9.7]. 
Proposition 1.19. Let $D \in Z_{l}\left(L_{n-k}^{n}\right)$ be a subcycle. Then

$$
D \cdot\left[L_{n-k}^{n}\right]=\left[L_{n-k}^{n}\right] \cdot D=D \quad \text { on } L_{n-k}^{n} .
$$

Proof. Let $\pi_{i}: L_{n-k}^{n} \times L_{n-k}^{n} \rightarrow L_{n-k}^{n}$ be the morphism given by $\left(x_{1}, x_{2}\right) \mapsto x_{i}$. By Remark 1.12,

$$
\begin{aligned}
D \cdot\left[L_{n-k}^{n}\right] & =\left(\pi_{1}\right)_{*}\left(\sum_{i=1}^{r} h_{i, 1} \cdots h_{i, n-k} \cdot\left(D \times\left[L_{n-k}^{n}\right]\right)\right) \\
& =\left(\pi_{1}\right)_{*}\left(\left(\sum_{i=1}^{r} h_{i, 1} \cdots h_{i, n-k}\right) \cdot\left(\left(\frac{0}{-e_{0}}\right)+\left(\frac{-e_{0}}{-e_{0}}\right)\right)^{k} \cdot\left(D \times \mathbb{R}^{n}\right)\right) \\
& =\left(\pi_{1}\right)_{*}\left(\triangle_{\mathbb{R}^{n}} \cdot\left(D \times \mathbb{R}^{n}\right)\right)=\left(\pi_{1}\right)_{*}\left(\triangle_{D}\right)=D .
\end{aligned}
$$

Furthermore, let $\phi: L_{k}^{n} \times L_{k}^{n} \rightarrow L_{k}^{n} \times L_{k}^{n}$ be the morphism induced by $(x, y) \mapsto(y, x)$. Obviously,

$$
\left(\sum_{i=1}^{r} h_{i, 1} \cdots h_{i, n-k}\right) \cdot\left[L_{n-k}^{n} \times L_{n-k}^{n}\right]=\left(\sum_{i=1}^{r} \phi^{*} h_{i, 1} \cdots \phi^{*} h_{i, n-k}\right) \cdot\left[L_{n-k}^{n} \times L_{n-k}^{n}\right] .
$$

If $\pi_{i j}:\left(L_{n-k}^{n}\right)^{4} \rightarrow\left(L_{n-k}^{n}\right)^{2}$ is the morphism given by $\left(x_{1}, x_{2}, x_{3}, x_{4}\right) \mapsto\left(x_{i}, x_{j}\right)$ and

$$
\triangle:=\left(\sum_{i=1}^{r} \pi_{13}^{*} h_{i, 1} \cdots \pi_{13}^{*} h_{i, n-k}\right) \cdot\left(\sum_{i=1}^{r} \pi_{24}^{*} h_{i, 1} \cdots \pi_{24}^{*} h_{i, n-k}\right)
$$

we conclude by [AR07, Proposition 7.7] and [AR07, Lemma 9.6] that

$$
\begin{aligned}
& \left(\sum_{i=1}^{r} \phi^{*} h_{i, 1} \cdots \phi^{*} h_{i, n-k}\right) \cdot\left(D \times\left[L_{n-k}^{n}\right]\right) \\
& =\left(\sum_{i=1}^{r} \phi^{*} h_{i, 1} \cdots \phi^{*} h_{i, n-k}\right) \cdot\left(\left(D \times\left[L_{n-k}^{n}\right]\right) \cdot\left(\left[L_{n-k}^{n} \times L_{n-k}^{n}\right]\right)\right) \\
& =\left(\sum_{i=1}^{r} \phi^{*} h_{i, 1} \cdots \phi^{*} h_{i, n-k}\right) \cdot\left(\pi_{12}\right)_{*}\left(\Delta \cdot\left(\left(D \times\left[L_{n-k}^{n}\right]\right) \times\left(\left[L_{n-k}^{n} \times L_{n-k}^{n}\right]\right)\right)\right) \\
& =\left(\sum_{i=1}^{r} \phi^{*} h_{i, 1} \cdots \phi^{*} h_{i, n-k}\right) \cdot\left(\pi_{12}\right)_{*}\left(\triangle_{D \times\left[L_{n-k}^{n}\right]}\right) \\
& =\left(\sum_{i=1}^{r} \phi^{*} h_{i, 1} \cdots \phi^{*} h_{i, n-k}\right) \cdot\left(\pi_{34}\right)_{*}\left(\triangle_{D \times\left[L_{n-k}^{n}\right]}\right) \\
& =\left(\pi_{34}\right)_{*}\left(\left(\sum_{i=1}^{r} \pi_{34}^{*} \phi^{*} h_{i, 1} \cdots \pi_{34}^{*} \phi^{*} h_{i, n-k}\right) \cdot \triangle \cdot\left(\left(D \times\left[L_{n-k}^{n}\right]\right) \times\left(\left[L_{n-k}^{n} \times L_{n-k}^{n}\right]\right)\right)\right)
\end{aligned}
$$




$$
\begin{aligned}
& =\left(\pi_{34}\right)_{*}\left(\Delta \cdot\left(D \times\left[L_{n-k}^{n}\right]\right) \times\left(\left(\sum_{i=1}^{r} \phi^{*} h_{i, 1} \cdots \phi^{*} h_{i, n-k}\right) \cdot\left[L_{n-k}^{n} \times L_{n-k}^{n}\right]\right)\right) \\
& =\left(\pi_{34}\right)_{*}\left(\Delta \cdot\left(D \times\left[L_{n-k}^{n}\right]\right) \times\left(\left(\sum_{i=1}^{r} h_{i, 1} \cdots h_{i, n-k}\right) \cdot\left[L_{n-k}^{n} \times L_{n-k}^{n}\right]\right)\right) \\
& =\left(\sum_{i=1}^{r} h_{i, 1} \cdots h_{i, n-k}\right) \cdot\left(D \times\left[L_{n-k}^{n}\right]\right) .
\end{aligned}
$$

Hence we deduce that

$$
\begin{aligned}
D \cdot\left[L_{n-k}^{n}\right] & =\left(\pi_{1}\right)_{*}\left(\triangle_{D}\right)=\left(\pi_{2}\right)_{*}\left(\triangle_{D}\right)=\left(\pi_{2}\right)_{*}\left(\left(\sum_{i=1}^{r} h_{i, 1} \cdots h_{i, n-k}\right) \cdot\left(D \times\left[L_{n-k}^{n}\right]\right)\right) \\
& =\left(\pi_{2}\right)_{*}\left(\left(\sum_{i=1}^{r} \phi^{*} h_{i, 1} \cdots \phi^{*} h_{i, n-k}\right) \cdot\left(D \times\left[L_{n-k}^{n}\right]\right)\right) \\
& =\left(\pi_{1}\right)_{*}\left(\left(\sum_{i=1}^{r} h_{i, 1} \cdots h_{i, n-k}\right) \cdot\left(\left[L_{n-k}^{n}\right] \times D\right)\right)=\left[L_{n-k}^{n}\right] \cdot D .
\end{aligned}
$$

Remark 1.20. We can prove in the same way that $\left[L_{n-k}^{n} \times L_{m-l}^{m}\right] \cdot D=D$ for all subcycles $D$ of $L_{n-k}^{n} \times L_{m-l}^{m}$ and even that $\left[L_{n_{1}-k_{1}}^{n_{1}} \times \cdots \times L_{n_{r}-k_{r}}^{n_{r}}\right] \cdot D=D$ for all $r \geq 1$ and all subcycles $D$ of $L_{n_{1}-k_{1}}^{n_{1}} \times \cdots \times L_{n_{r}-k_{r}}^{n_{r}}$. Moreover, restricting the intersection products to open subsets of $\left|L_{k}^{n}\right|$ or $\left|L_{n_{1}-k_{1}}^{n_{1}} \times \cdots \times L_{n_{r}-k_{r}}^{n_{r}}\right|$, respectively, implies that $X \cdot D=D$ for all subcycles $D \in Z_{l}(X)$ if $X \in\left\{[\widetilde{F}],\left[\widetilde{F}_{1} \times \cdots \times \widetilde{F}_{r}\right]\right\}$ where $\widetilde{F}, \widetilde{F}_{i}$ are tropical fans associated to open subsets of some $\left|L_{k}^{n}\right|$ as in Corollary 1.13.

Proposition 1.21. Let $C \in Z_{k}\left(\mathbb{R}^{n}\right)$ be a tropical cycle that admits an intersection product as in Definition 1.16 and let $D, D^{\prime} \in Z_{l}(C), E \in Z_{l^{\prime}}(C)$ be subcycles. Then

$$
\left(D+D^{\prime}\right) \cdot E=D \cdot E+D^{\prime} \cdot E .
$$

Proof. The proof is exactly the same as for [AR07, Theorem 9.10(b)].

Proposition 1.22. Let $C \in Z_{k}\left(\mathbb{R}^{n}\right)$ be a tropical cycle that admits an intersection product as in Definition 1.16 and let $D \in Z_{l}(C)$ be a subcycle of $C$. Moreover, let $E \in Z_{l^{\prime}}(C)$ be a subcycle such that there are Cartier divisors $\psi_{i, j} \in \operatorname{Div}(C)$ with

$$
\sum_{i=1}^{r} \psi_{i, 1} \cdots \psi_{i, k-l^{\prime}} \cdot C=E .
$$

If additionally $C \cdot D=D$ then

$$
\sum_{i=1}^{r} \psi_{i, 1} \cdots \psi_{i, k-l^{\prime}} \cdot D=E \cdot D .
$$

Proof. The proof is the same as for [AR07, Corollary 9.8]. 
Remark 1.23. The meaning of Proposition 1.22 is the following: If $X \in Z_{k}\left(\mathbb{R}^{n}\right)$ is a tropical cycle such that the diagonal $\triangle_{X}$ can be written as a sum of products of Cartier divisors as in Definition 1.16 and additionally $(X \times X) \cdot Y=Y$ for all subcycles $Y$ of $X \times X$ then we can apply Proposition 1.22 with $C:=X \times X$ and $E:=\triangle_{X}$ to deduce that the definition of the intersection product is independent of the choice of the Cartier divisors describing the diagonal. In particular we have well-defined intersection products on $L_{k}^{n}, L_{k_{1}}^{n_{1}} \times \cdots \times L_{k_{r}}^{n_{r}}, \widetilde{F}$ and $\widetilde{F}_{1} \times \cdots \times \widetilde{F}_{r}$ for all tropical fans $\widetilde{F}, \widetilde{F}_{i}$ associated to an open subset of some $\left|L_{k}^{n}\right|$ as in Corollary 1.13 .

Theorem 1.24. Let $C \in Z_{k}\left(\mathbb{R}^{n}\right)$ be a tropical cycle that admits an intersection product as in Definition 1.16 such that additionally $(C \times C) \cdot D=D$ for all subcycles $D$ of $C \times C$. Moreover, let $E, E^{\prime} \in Z_{l}(C), F \in Z_{l^{\prime}}(C)$ and $G \in Z_{l^{\prime \prime}}(C)$ be subcycles. Then

(a) $E \cdot F=F \cdot E$,

(b) $(E \cdot F) \cdot G=E \cdot(F \cdot G)$.

Proof. The proof is exactly the same as for [AR07, Theorem 9.10 (a)\&(c)].

We finish this section with an example showing that even curves intersecting in the expected dimension can have negative intersections:

Example 1.25. Let $C, D \in Z_{1}\left(L_{2}^{3}\right)$ be the curves shown in the figure. We want to compute the intersection $C \cdot D$. By Proposition 1.22 the easiest way to do this is to write one of the curves as $\psi \cdot\left[L_{2}^{3}\right]$ for some Cartier divisor $\psi$ on $L_{2}^{3}$.

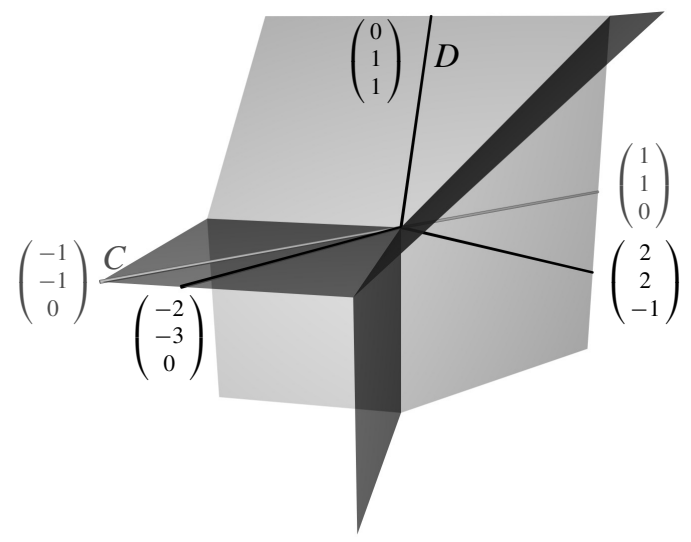

Let $F$ be the refinement of $L_{2}^{3}$ arising by dividing the cones $\left\langle-e_{1},-e_{2}\right\rangle_{\mathbb{R}_{\geq 0}}$ and $\left\langle-e_{0},-e_{3}\right\rangle_{\mathbb{R}_{\geq 0}}$ into the cones $\left\langle-e_{1},-e_{1}-e_{2}\right\rangle_{\mathbb{R}_{\geq 0}},\left\langle-e_{2},-e_{1}-e_{2}\right\rangle_{\mathbb{R}_{\geq 0}}$ and $\left\langle-e_{0},-e_{0}-\right.$ $\left.e_{3}\right\rangle_{\mathbb{R}_{\geq 0}},\left\langle-e_{3},-e_{0}-e_{3}\right\rangle_{\mathbb{R}_{\geq 0}}$, respectively. Then

$$
\psi:=\left(\begin{array}{l}
1 \\
1 \\
1
\end{array}\right)-\left(\begin{array}{c}
-1 \\
-1 \\
0
\end{array}\right)
$$

defines a rational function on $F$. As shown in [AR07, Example 3.10] we have $\psi \cdot\left[L_{2}^{3}\right]=C$. 
Hence we calculate

$$
\begin{aligned}
C \cdot D=\psi \cdot D & =\left(\psi\left(\begin{array}{c}
-2 \\
-3 \\
0
\end{array}\right)+\psi\left(\begin{array}{l}
2 \\
2 \\
1
\end{array}\right)+\psi\left(\begin{array}{l}
0 \\
1 \\
1
\end{array}\right)-\psi\left(\begin{array}{l}
0 \\
0 \\
0
\end{array}\right)\right) \cdot\{0\} \\
& =(-2+0+1-0) \cdot\{0\}=-1 \cdot\{0\} .
\end{aligned}
$$

Remark 1.26. This result is remarkable for the following reason: Our ambient space $L_{2}^{3}$ arises as a so-called modification of $\mathbb{R}^{2}$ (cf. [M06], [M07]). Varieties that are connected by a series of modifications are called equivalent by G. Mikhalkin and are expected to have similar properties. But the above example shows that there is a big difference between $\mathbb{R}^{2}$ and $L_{2}^{3}$ even though they are equivalent: On $\mathbb{R}^{2}$ there is no negative intersection product of curves, on $L_{2}^{3}$ there is.

\section{Intersection products on smooth tropical varieties}

In this section we use our results from Section 1 to define an intersection product on smooth tropical varieties, i.e. on varieties with tropical linear spaces as local building blocks:

Definition 2.1 (Smooth tropical varieties). An abstract tropical variety $C$ is called a smooth variety if it has a representative $\left(\left((X,|X|), \omega_{X}\right),\left\{\Phi_{\sigma}\right\}\right)$ such that all the maps

$$
\Phi_{\sigma}: S_{\sigma}=\bigcup_{\sigma^{\prime} \in X^{*}, \sigma^{\prime} \supset \sigma}\left(\sigma^{\prime}\right)^{r i} \stackrel{\sim}{\rightarrow}\left|F_{\sigma}\right| \subseteq\left|\widetilde{F}_{\sigma}\right|
$$

(cf. [AR07, Definition 5.4]) map into tropical fans $\widetilde{F_{\sigma}}=\widetilde{F_{1}^{\sigma}} \times \cdots \times \widetilde{F_{r_{\sigma}}^{\sigma}}$ where the $\widetilde{F_{i}^{\sigma}}$ are tropical fans associated to open subsets of some $\left|L_{k_{\sigma, i}}^{n_{\sigma, i}}\right|$ as in Corollary 1.13.

Remark 2.2. Note that the existence of such a representative $\left(\left((X,|X|), \omega_{X}\right),\left\{\Phi_{\sigma}\right\}\right)$ for $C$ implies that all representatives of $C$ have the required property.

Example 2.3. The following figures show two examples of smooth tropical varieties:
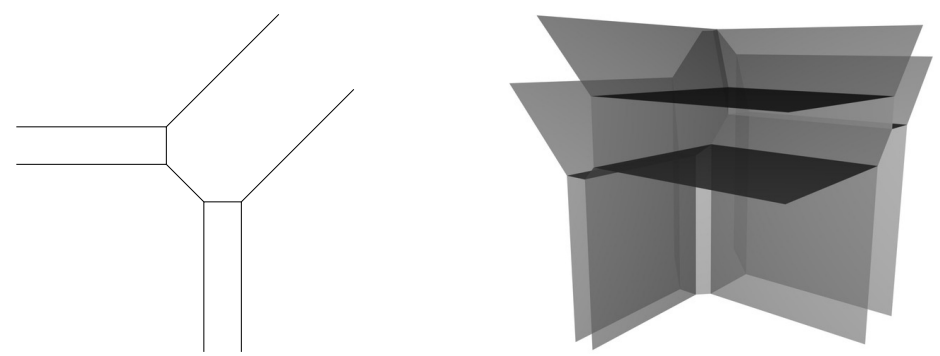
Definition 2.4. Let $C$ be an abstract tropical cycle, $D$ a subcycle of $C$ with representative $X$ and $U \subseteq|C|$ an open subset. We denote by $X \cap U$ the open tropical polyhedral complex

$$
X \cap U:=(\{\sigma \cap U \mid \sigma \in X\},|X| \cap U)
$$

and by $[X \cap U]$ its equivalence class modulo refinements. As this class only depends on the class of $X$ we can define $D \cap U:=[X \cap U]$.

Remark 2.5. If we are given an open covering $\left\{U_{1}, \ldots, U_{r}\right\}$ of $C$ and open tropical polyhedral complexes $D_{1} \cap U_{1}, \ldots, D_{r} \cap U_{r}$ such that $D_{i} \cap U_{i} \cap U_{j}=D_{j} \cap U_{i} \cap U_{j}$ we can glue $D_{1} \cap U_{1}, \ldots, D_{r} \cap U_{r}$ to obtain a cycle $D \in Z_{*}(C)$.

Definition 2.6 (Intersection products). Let $C$ be a smooth tropical variety and let $\left(\left((X,|X|), \omega_{X}\right),\left\{\Phi_{\sigma}\right\}\right)$ be a representative of $C$ as in Definition 2.1. Moreover, let $D, E$ be subcycles of $C$. We construct local intersection products as follows: For every $\sigma \in X$ we can regard $\left(D \cap S_{\sigma}\right)$ and $\left(E \cap S_{\sigma}\right)$ as open tropical cycles in $\widetilde{F}_{\sigma}$ via the map $\Phi_{\sigma}$. Let $\widetilde{D \cap S_{\sigma}}$ and $\widehat{E \cap S_{\sigma}}$ be any tropical cycles in $\widetilde{F}_{\sigma}$ restricting to $D \cap S_{\sigma}$ and $E \cap S_{\sigma}$. As we have an intersection product on $\widetilde{F}_{\sigma}$ by Remark 1.23 we can define the intersection

$$
\left(D \cdot_{\sigma} E\right) \cap S_{\sigma}:=\left(\left(\widetilde{D \cap S_{\sigma}}\right) \cdot\left(\widetilde{E \cap S_{\sigma}}\right)\right) \cap S_{\sigma} .
$$

Note that $\left(D \cdot_{\sigma} E\right) \cap S_{\sigma}$ does not depend on the choice of the cycles $\widetilde{D \cap S_{\sigma}}$ and $\widetilde{E \cap S_{\sigma}}$. Since $\left\{S_{\sigma} \mid \sigma \in X\right\}$ is an open covering of $|C|$ and the local intersection products $\left(D \cdot{ }_{\sigma} E\right) \cap S_{\sigma}, \sigma \in X$, are compatible by the lemma below, we can glue them to obtain a global intersection cycle $D \cdot E \in Z_{*}(C)$.

Lemma 2.7. For the local intersection products in Definition 2.6 we have

$$
(D \cdot \sigma E) \cap S_{\sigma} \cap S_{\sigma^{\prime}}=\left(D \cdot \sigma^{\prime} E\right) \cap S_{\sigma} \cap S_{\sigma^{\prime}} .
$$

Proof. By definition we have an integer linear map

$$
\left|\widetilde{F}_{1}\right| \supseteq \Phi_{\sigma}\left(S_{\sigma} \cap S_{\sigma^{\prime}}\right) \stackrel{f}{\rightarrow} \Phi_{\sigma^{\prime}}\left(S_{\sigma} \cap S_{\sigma^{\prime}}\right) \subseteq\left|\widetilde{F}_{2}\right|
$$

with integer linear inverse $f^{-1}$, where $\widetilde{F}_{1}, \widetilde{F}_{2}$ are the tropical fans generated by $\Phi_{\sigma}\left(S_{\sigma} \cap S_{\sigma^{\prime}}\right)$ and $\Phi_{\sigma^{\prime}}\left(S_{\sigma} \cap S_{\sigma^{\prime}}\right)$, respectively. Let $C_{1}, C_{2}$ be subcycles of $\widetilde{F}_{1}$. We have to show that

$$
C_{1} \cdot C_{2}=\left(f^{-1}\right)_{*}\left(f_{*}\left(C_{1}\right) \cdot f_{*}\left(C_{2}\right)\right) .
$$

If $\pi$ is the respective projection on the first factor we obtain, by Proposition 1.22 and Remark 1.23,

$$
\begin{aligned}
\left(f^{-1}\right)_{*}\left(f_{*}\left(C_{1}\right) \cdot f_{*}\left(C_{2}\right)\right) & =\left(f^{-1}\right)_{*}\left(\pi_{*}\left(\triangle_{\tilde{F}_{2}} \cdot\left(f_{*}\left(C_{1}\right) \times f_{*}\left(C_{2}\right)\right)\right)\right) \\
& =\pi_{*}\left(\left(f^{-1} \times f^{-1}\right)_{*}\left(\triangle_{\widetilde{F}_{2}} \cdot\left(f_{*}\left(C_{1}\right) \times f_{*}\left(C_{2}\right)\right)\right)\right) \\
& =\pi_{*}\left(\left(f^{-1} \times f^{-1}\right)_{*}\left((f \times f)_{*}\left(\triangle_{\tilde{F}_{1}}\right) \cdot(f \times f)_{*}\left(C_{1} \times C_{2}\right)\right)\right) \\
& =\pi_{*}\left(\triangle_{\widetilde{F}_{1}} \cdot C_{1} \times C_{2}\right)=C_{1} \cdot C_{2} .
\end{aligned}
$$


Remark 2.8. Lemma 2.7 also implies that further refinements of the representative $\left(\left((X,|X|), \omega_{X}\right),\left\{\Phi_{\sigma}\right\}\right)$ of $C$ do not change the result $D \cdot E$. Hence the intersection product is well-defined.

Our last step consists in proving basic properties of our intersection product:

Theorem 2.9. Let $C$ be a smooth tropical variety, let $D, D^{\prime} \in Z_{l}(C), E \in Z_{l^{\prime}}(C)$ and $F \in Z_{l^{\prime \prime}}(C)$ be subcycles and let $\varphi \in \operatorname{Div}(C)$ be a Cartier divisor on $C$. Then the following equalities hold in $Z_{*}(C)$ :

(a) $C \cdot D=D$,

(b) $D \cdot E=E \cdot D$,

(c) $\left(D+D^{\prime}\right) \cdot E=D \cdot E+D^{\prime} \cdot E$,

(d) $(D \cdot E) \cdot F=D \cdot(E \cdot F)$,

(e) $\varphi \cdot(D \cdot E)=(\varphi \cdot D) \cdot E$.

If moreover $D=\left(\sum_{i=1}^{r} \varphi_{i, 1} \cdots \varphi_{i, l}\right) \cdot C$ for some Cartier divisors $\varphi_{i, j} \in \operatorname{Div}(C)$ then

$$
D \cdot E=\sum_{i=1}^{r} \varphi_{i, 1} \cdots \varphi_{i, l} \cdot E
$$

Proof. The statements follow immediately from the definition of the intersection product and the corresponding statements in Section 1.

\section{Pull-backs of cycles on smooth varieties}

We will now use the intersection product defined in Section 2 to introduce pull-backs of tropical cycles along morphisms between smooth tropical varieties.

Definition 3.1 (Pull-back). Let $X$ and $Y$ be smooth tropical varieties of dimension $m$ and $n$, respectively, and let $f: X \rightarrow Y$ be a morphism of tropical cycles. Moreover, let $\pi: X \times Y \rightarrow X$ be the projection onto the first factor and let $\gamma_{f}: X \rightarrow X \times Y$ be the morphism given by $x \mapsto(x, f(x))$. We denote by $\Gamma_{f}:=\left(\gamma_{f}\right)_{*} X$ the graph of $f$. For a cycle $C \in Z_{n-k}(Y)$ we define its pull-back $f^{*} C \in Z_{m-k}(X)$ to be

$$
f^{*} C:=\pi_{*}\left(\Gamma_{f} \cdot(X \times C)\right) .
$$

The easiest non-trivial, but nevertheless important example of a pull-back is the following:

Example 3.2. Let $C$ and $D$ be smooth tropical cycles and let $p: C \times D \rightarrow D$ be the projection on the second factor. We want to calculate the pull-back $p^{*} E$ for a cycle $E \in Z_{k}(D)$ : The map $\gamma_{p}$ from Definition 3.1 is then just given by $\gamma_{p}: C \times D \rightarrow$ $C \times D \times D:(x, y) \mapsto(x, y, y)$ and the map $\pi: C \times D \times D \rightarrow C \times D$ is the projection to the first two factors. Hence we can conclude that $\Gamma_{p}=C \times \Delta_{D}$. Moreover, 
let $\pi^{1}: C \times D \times D \rightarrow C$ be the projection to the first factor and $\pi^{2}: C \times D \times D \rightarrow D$ be the projection to the second factor. We obtain, by Definition 3.1,

$$
\begin{aligned}
p^{*} E & =\pi_{*}\left(\Gamma_{p} \cdot(C \times D \times E)\right)=\pi_{*}\left(\left(C \times \triangle_{D}\right) \cdot(C \times D \times E)\right) \\
& =\pi_{*}^{1}(C \cdot C) \times \pi_{*}^{2}\left(\triangle_{D} \cdot(D \times E)\right)=C \times E .
\end{aligned}
$$

The pull-back has the following basic properties:

Theorem 3.3. Let $X, Y$ and $Z$ be smooth tropical varieties and let $f: X \rightarrow Y$ and $g$ : $Y \rightarrow Z$ be morphisms of tropical cycles. Moreover, let $C, C^{\prime} \in Z_{*}(Y)$ and $D \in Z_{*}(X)$ be subcycles. Then

(a) $f^{*} Y=X$,

(b) $\operatorname{id}_{Y}^{*} C=C$,

(c) if $C=\varphi_{1} \cdots \varphi_{r} \cdot Y$ then $f^{*} C=f^{*} \varphi_{1} \cdots f^{*} \varphi_{r} \cdot X$,

(d) $C \cdot f_{*} D=f_{*}\left(f^{*} C \cdot D\right)$,

(e) $(g \circ f)^{*} C=f^{*} g^{*} C$,

(f) $f^{*}\left(C \cdot C^{\prime}\right)=f^{*} C \cdot f^{*} C^{\prime}$.

Proof. Throughout the proof, let $\pi^{X}, \pi_{X}, \pi^{1}, \pi_{1}, \pi^{Y}, \pi_{Y}, \pi^{2}, \pi_{2}, \pi^{X, Y}, \pi_{X, Y}, \pi^{1,2}$, $\pi_{1,2}$ and so forth be the projections to the respective factors.

(a)\&(b) By the definition of pull-back

$$
f^{*} Y=\pi_{*}^{X}\left(\Gamma_{f} \cdot(X \times Y)\right)=\pi_{*}^{X}\left(\Gamma_{f}\right)=X
$$

and

$$
\operatorname{id}_{Y}^{*} C=\pi_{*}^{1}\left(\Gamma_{\mathrm{id}_{Y}} \cdot(Y \times C)\right)=\pi_{*}^{1}\left(\triangle_{Y} \cdot(Y \times C)\right)=Y \cdot C=C .
$$

(c) We have

$$
\begin{aligned}
f^{*} C & =\pi_{*}^{X}\left(\Gamma_{f} \cdot\left(X \times\left(\varphi_{1} \cdots \varphi_{r} \cdot Y\right)\right)\right)=\pi_{*}^{X}\left(\pi_{2}^{*} \varphi_{1} \cdots \pi_{2}^{*} \varphi_{r} \cdot \Gamma_{f} \cdot(X \times Y)\right) \\
& =\pi_{*}^{X}\left(\pi_{2}^{*} \varphi_{1} \cdots \pi_{2}^{*} \varphi_{r} \cdot \Gamma_{f}\right) .
\end{aligned}
$$

By the definition of intersection product (see [AR07, Definitions 3.4 and 6.5]) this last line is equal to

$$
f^{*} \varphi_{1} \cdots f^{*} \varphi_{r} \cdot X
$$

(d) Let $\pi_{X}: X \times Y \rightarrow X$ be the projection on $X$. By Example 3.2 we know that $\pi_{X}^{*} D=D \times Y$. As the diagonal $\triangle_{X}$ can locally be expressed by Cartier divisors we can apply [AR07, Proposition 7.7] and statement (c) locally to deduce that for all subcycles $E$ of $X \times Y$,

$$
\begin{aligned}
D \cdot \pi_{*}^{X} E & =\pi_{*}^{1}\left(\triangle_{X} \cdot\left(D \times \pi_{*}^{X} E\right)\right)=\pi_{*}^{1}\left(\triangle_{X} \cdot\left(\mathrm{id} \times \pi^{X}\right)_{*}(D \times E)\right) \\
& =\pi_{*}^{1}\left(\left(\mathrm{id} \times \pi^{X}\right)_{*}\left(\left(\mathrm{id} \times \pi^{X}\right)^{*} \triangle_{X} \cdot(D \times E)\right)\right) \\
& =\pi_{*}^{1}\left(\left(\mathrm{id} \times \pi^{X}\right)_{*}\left(\left(\triangle_{X} \times Y\right) \cdot(D \times E)\right)\right) \\
& =\pi_{*}^{1}\left(\pi_{*}^{1,2}\left(\left(\triangle_{X} \times Y\right) \cdot(D \times E)\right)\right) \\
& =\pi_{*}^{1}\left(\pi_{*}^{1,2}\left(\triangle_{X \times Y} \cdot(D \times Y \times E)\right)\right)=\pi_{*}^{1}((D \times Y) \cdot E)=\pi_{*}^{X}\left(\pi_{X}^{*} D \cdot E\right) .
\end{aligned}
$$


This implies that

$$
\begin{aligned}
f^{*} C \cdot D & =D \cdot \pi_{*}^{X}\left(\Gamma_{f} \cdot(X \times C)\right)=\pi_{*}^{X}\left(\pi_{X}^{*} D \cdot \Gamma_{f} \cdot(X \times C)\right) \\
& =\pi_{*}^{X}\left((D \times Y) \cdot \Gamma_{f} \cdot(X \times C)\right)=\pi_{*}^{X}\left(\Gamma_{f} \cdot(D \times C)\right) .
\end{aligned}
$$

Moreover, it is easy to check that $(f \times \text { id })^{*} \triangle_{Y}=\Gamma_{f}$. As above we conclude that

$$
\begin{aligned}
C \cdot f_{*} D & =\pi_{*}^{1}\left(\triangle_{Y} \cdot\left(C \times f_{*} D\right)\right)=\pi_{*}^{1}\left((\mathrm{id} \times f)_{*}\left((\mathrm{id} \times f)^{*} \triangle_{Y} \cdot(C \times D)\right)\right) \\
& =f_{*}\left(\pi_{*}^{X}\left((\mathrm{id} \times f)^{*} \triangle_{Y} \cdot(C \times D)\right)\right)=f_{*}\left(\pi_{*}^{X}\left((f \times \mathrm{id})^{*} \triangle_{Y} \cdot(D \times C)\right)\right) \\
& =f_{*}\left(\pi_{*}^{X}\left(\Gamma_{f} \cdot(D \times C)\right)\right)=f_{*}\left(f^{*} C \cdot D\right) .
\end{aligned}
$$

(e) Let $\Phi: X \rightarrow X \times Y \times Z$ be given by $x \mapsto(x, f(x), g(f(x)))$. An easy calculation shows that $\left(\Gamma_{f} \times Z\right) \cdot\left(X \times \Gamma_{g}\right)=\Phi_{*} X$. Hence we conclude by (d) that

$$
\begin{aligned}
f^{*} g^{*} C & =\pi_{*}^{X}\left(\Gamma_{f} \cdot\left(X \times \pi_{*}^{Y}\left(\Gamma_{g} \cdot(Y \times C)\right)\right)\right) \\
& =\pi_{*}^{X}\left(\pi_{*}^{X, Y}\left(\left(\Gamma_{f} \times Z\right) \cdot\left(X \times \Gamma_{g}\right) \cdot(X \times Y \times C)\right)\right) \\
& =\pi_{*}^{X}\left(\left(\Gamma_{f} \times Z\right) \cdot\left(X \times \Gamma_{g}\right) \cdot(X \times Y \times C)\right) \\
& =\pi_{*}^{X}\left(\Phi_{*} X \cdot(X \times Y \times C)\right)=\pi_{*}^{X}\left(\Gamma_{g \circ f} \cdot(X \times C)\right)=(g \circ f)^{*} C .
\end{aligned}
$$

(f) Let $\Phi: X \rightarrow X \times Y \times Y$ be given by $x \mapsto(x, f(x), f(x))$ and let $\pi^{1,2}, \pi^{1,3}$ : $X \times Y \times Y \rightarrow X \times Y$ be the projections to the respective factors. An easy calculation shows that

$$
\left(\Gamma_{f} \times Y\right) \cdot\left(X \times \Gamma_{\mathrm{id}_{Y}}\right)=\Phi_{*} X=\pi_{1,2}^{*} \Gamma_{f} \cdot \pi_{1,3}^{*} \Gamma_{f} .
$$

Hence we deduce that

$$
\begin{aligned}
f^{*}\left(C \cdot C^{\prime}\right) & =\pi_{*}^{X}\left(\Gamma_{f} \cdot\left(X \times\left(C \cdot C^{\prime}\right)\right)\right)=\pi_{*}^{X}\left(\Gamma_{f} \cdot\left(X \times \pi_{*}^{1}\left(\Gamma_{\mathrm{id}_{Y}} \cdot C \times C^{\prime}\right)\right)\right) \\
& =\pi_{*}^{X}\left(\Gamma_{f} \cdot \pi_{*}^{1,2}\left(\left(X \times \Gamma_{\mathrm{id} Y}\right) \cdot\left(X \times C \times C^{\prime}\right)\right)\right) \\
& =\pi_{*}^{X}\left(\pi_{*}^{1,2}\left(\left(\Gamma_{f} \times Y\right) \cdot\left(X \times \Gamma_{\mathrm{id}_{Y}}\right) \cdot\left(X \times C \times C^{\prime}\right)\right)\right) \\
& =\pi_{*}^{X}\left(\pi_{*}^{1,3}\left(\left(\Gamma_{f} \times Y\right) \cdot\left(X \times \Gamma_{\mathrm{id}_{Y}}\right) \cdot\left(X \times C \times C^{\prime}\right)\right)\right) \\
& =\pi_{*}^{X}\left(\pi_{*}^{1,3}\left(\pi_{1,2}^{*} \Gamma_{f} \cdot \pi_{1,3}^{*} \Gamma_{f} \cdot\left(X \times C \times C^{\prime}\right)\right)\right) \\
& =\pi_{*}^{X}\left(\Gamma_{f} \cdot \pi_{*}^{1,3}\left(\left(\Gamma_{f} \times Y\right) \cdot\left(X \times C \times C^{\prime}\right)\right)\right) \\
& =\pi_{*}^{X}\left(\Gamma_{f} \cdot\left(\pi_{*}^{X}\left(\Gamma_{f} \cdot(X \times C)\right) \times C^{\prime}\right)\right) \\
& =\pi_{*}^{X}\left(\Gamma_{f} \cdot\left(f^{*} C \times C^{\prime}\right)\right)=f^{*} C \cdot f^{*} C^{\prime} .
\end{aligned}
$$

We finish the section with another important example:

Example 3.4. Let $D$ be a smooth tropical variety and let $C \in Z_{k}(D)$ be a smooth tropical subvariety. Moreover, let $\iota: C \rightarrow D$ be the inclusion map. We want to calculate the pullback $\iota^{*} E$ for a cycle $E \in Z_{l}(D)$ : Let $\pi^{C}: C \times D \rightarrow C$ and $\pi^{D}: C \times D \rightarrow D$ be the projections to the first and second factor and let $\gamma_{\iota}: C \rightarrow C \times D$ be given by $x \mapsto(x, x)$. 
Hence, $\Gamma_{\iota}=\left(\gamma_{\iota}\right)_{*} C=\Delta_{C}$ and by Example 3.2, $\left(\pi^{D}\right)^{*} E=C \times E$. Thus by Theorem $3.3(d)$

$$
\begin{aligned}
\iota^{*} E & =\pi_{*}^{C}\left(\Gamma_{\iota} \cdot(C \times E)\right)=\pi_{*}^{C}\left(\triangle_{C} \cdot(C \times E)\right)=\pi_{*}^{D}\left(\triangle_{C} \cdot(C \times E)\right) \\
& =\pi_{*}^{D}\left(\triangle_{C} \cdot\left(\pi^{D}\right)^{*} E\right)=\pi_{*}^{D}\left(\triangle_{C}\right) \cdot E=C \cdot E,
\end{aligned}
$$

where $C \cdot E$ is the intersection product on $D$.

Acknowledgments. I would like to thank my advisor Andreas Gathmann for numerous helpful discussions.

\section{References}

[AR07] Allermann, L., Rau, J.: First steps in tropical intersection theory. Math. Z. 264, 633-670 (2010) Zbl 1193.14074 MR 2591823

[AR08] Allermann, L., Rau, J.: Tropical rational equivalence. arXiv:0811.2860

[GKM07] Gathmann, A., Kerber, M., Markwig, H.: Tropical fans and the moduli spaces of tropical curves. Compos. Math. 145, 173-195 (2009) Zbl 1169.51021 MR 2480499

[M06] Mikhalkin, G.: Tropical geometry and its applications. In: Proc. ICM (Madrid, 2006), 827-852 Zbl 1103.14034 MR 2275625

[M07] Mikhalkin, G.: Introduction to tropical geometry. In: Notes from the IMPA lectures (2007), arXiv:0709.1049 